\title{
Measure of bubble non-uniformity within circular region in a direct-contact heat exchanger
}

DOI:

10.1016/j.ijheatmasstransfer.2017.03.042

\section{Document Version}

Accepted author manuscript

Link to publication record in Manchester Research Explorer

\section{Citation for published version (APA):}

Xiao, Q., Pan, J., Lv, Z., Xu, J., \& Wang, H. (2017). Measure of bubble non-uniformity within circular region in a direct-contact heat exchanger. International Journal of Heat and Mass Transfer, 110, 257-261.

https://doi.org/10.1016/j.ijheatmasstransfer.2017.03.042

\section{Published in:}

International Journal of Heat and Mass Transfer

\section{Citing this paper}

Please note that where the full-text provided on Manchester Research Explorer is the Author Accepted Manuscript or Proof version this may differ from the final Published version. If citing, it is advised that you check and use the publisher's definitive version.

\section{General rights}

Copyright and moral rights for the publications made accessible in the Research Explorer are retained by the authors and/or other copyright owners and it is a condition of accessing publications that users recognise and abide by the legal requirements associated with these rights.

\section{Takedown policy}

If you believe that this document breaches copyright please refer to the University of Manchester's Takedown Procedures [http://man.ac.uk/04Y6Bo] or contact uml.scholarlycommunications@manchester.ac.uk providing relevant details, so we can investigate your claim.

\section{OPEN ACCESS}




\title{
Measure of bubble non-uniformity within circular region in a direct-contact heat exchanger
}

\author{
Qingtai Xiao ${ }^{\mathrm{a}, \mathrm{b}, 1}$, Jianxin $\mathrm{Pan}^{\mathrm{c}, 1}$, Zhihan $\mathrm{Lv}^{\mathrm{d}}$, Jianxin $\mathrm{Xu}^{a, e, *}, \mathrm{Hua} \mathrm{Wang}^{a, b}$ \\ ${ }^{a}$ State Key Laboratory of Complex Nonferrous Metal Resources Clean Utilization, Kunming University of Science and Technology, Kunming \\ 650093, PR China \\ ${ }^{b}$ Faulty of Metallurgy and Energy Engineering, Kunming University of Science and Technology, Kunming 650093, PR China \\ ${ }^{c}$ School of Mathematics, University of Manchester, Manchester M13 9PL, UK \\ ${ }^{d}$ Department of Computer Science, University College London, London WC1E 6EA, UK \\ ${ }^{e}$ Quality Development Institute, Kunming University of Science and Technology, Kunming 650093, PR China
}

\begin{abstract}
The study of bubble swarm distributions in a direct-contact heat exchanger was addressed both theoretically and experimentally. But recently developed approach based on uniform design for measuring bubbles uniformity and mixing efficiency within rectangular or square region can not be used to characterise the mixture homogeneity and mixing time within circular region. This paper discussed the difference between rectangular or square region and circular region. A quantitative metric of mixing uniformity is used to characterize the bubble patterns within circular region. Simulation studies are also used to illustrate the proposed methodologies while the experimental case considered demonstrate that the proposed approach evaluated the experimental data rather well. The space-time features of the mixing transient have been successfully derived when the bubble swarms or other objects distribute in a circular region. This work brings new insights to evaluate mixing quality of different systems practically, which has a high degree of accuracy (the percentage of enhancing achieved is about $40 \%$ ).
\end{abstract}

Keywords: bubble swarm; distribution uniformity; circular region; discrepancy method; direct-contact heat transfer.

\section{Introduction}

Direct-contact heat exchanger (DCHE) is one of the most efficient kinds of heat exchangers widely used in numerous engineering systems for carrying out heat transfer between two immiscible fluids[1]. It presents an alternative route for efficient utilization of low temperature waste heat resources. The heat transfer between the phases takes place at the 5 interface. Therefore the knowledge of heat transfer enhancement is fundamental for the design and operation of DCHE[2]. And it is necessary to know about the heat transfer characteristics of dispersed phase droplets in a continuous medium. Due to the complexity of the multiphase flow in exchangers, the heat exchange performance has often been expressed in terms of the volumetric heat transfer coefficient [3]. Huang et al. (2014) reported that homogeneous regime is one of the basic bubbling regimes in DCHE but less likely to prevail industrially owing to the high gas flow rates employed. This favors the 10 heterogeneous bubbling regime characterized by a wide range of bubble sizes and considerable frequencies of breakage and coalescence 4. Furthermore, the bubble uniformity and mixing efficiency are quantities related to the direct-contact heat transfer coefficient in the light of computational homology [5]. It was found that the direct-contact heat transfer performance was not only about the number of bubble swarms which could be characterized by Betti numbers, but it was all about the spatial-time location of bubble swarms because aggregate was considered to be related to the specific surface area $[6$. Mixing must satisfy not only the needs of mass and heat transfer but also the required homogeneity in the vessel in the shortest time

\footnotetext{
${ }^{1}$ These authors have contributed equally to this work.

*Corresponding author. Tel./Fax.: +86-0871-65157341.

E-mail addresses: xujianxina@163.com \& jianxinxu@kmust.edu.cn (Jianxin Xu); wanghua65@163.com (Hua Wang).
} 
in order to obtain high-quality products and high efficiency processes[7. Measuring the homogeneity properly is of great significant from the practical point view and for the validation of theoretical models as well 8 . The purpose of mixing is to obtain a homogeneous mixture. There is an increased desire for measuring and comparing mixing performance. The presence of dispersed phase makes the flow and mixing process of the continuous phase even complicated. Hence, the investigation of 20 characterizing the homogeneous bubbling regime is one of the most useful and instructive topics in DCHE.

Moreover, accurate characterization of fields uniformity is essential for the optimization of velocity field [9, 10, 11], electric fields 12, 13, magnetic fields 14, 15], etc.. A large number of mathematical works have been devoted to studying this issue. In practice, characterizing the mixing distribution of these flow patterns quantitatively using image processing technology, which has been effectively used for feature extraction in medical, bioengineering [16, pharmaceutics [17] and chemical industries, has drawn considerable interest in recent years. Aubin et al. (2010) reviewed existing methods for the characterisation of mixing and flow in microchannels, micromixers and microreactors [18. Gameiro et al. (2005) proposed the use of computational algebraic topology to obtain a characterization of the geometry of the patterns, produced by phase separation processes in compound materials 19. Xu et al. (2011) also applied computational algebraic topology and image analysis to quantify the mixture homogeneity and non-homogeneity in gas agitated rectors stirred by top lance gas injection 20]. The critical point determination of pseudo homogeneous process impact on the accurate estimation of mixing time could be investigated by three-sigma $(3 \sigma)$ method 2 . Fei et al. (2015) and Xu et al. (2016) addressed the key issue that how to measure the spacetime uniformity of random bubble swarm in a domain with minimum discrepancy [6, 21]. $S^{2}$, a more meaningful parameter, was also represented to study the mixing transient 22]. Of all the above studies, the $L_{2}$-star discrepancy method is one of the most valuable methods to determine mixing time and acquire more information of mixing process, which can successfully 35 quantify mixing time and degree of homogeneity.

The star discrepancy is the most well-known measure for the uniformity of point distributions 223, 24, 25, 26]. It is widely used in multivariate integration, pseudorandom number generation, statistics, and computer graphics. Particularly, regarding the measure of distribution uniformity of bubble swarm in direct-contact boiling heat transfer process, it is common practice to quantify the bubble swarm in rectangle region 6 , 21, 22]. However, the patterns were imaged through a circular viewing

40 window from where we could observe the most active stage of the bubbling regime in the bubble evaporation process. In addition, Fang and Wang (1994) found that if $B_{2}=\left\{(x, y): x^{2}+y^{2} \leq 1\right\}$ is a unit disk, and the natural method is using a circumscribed square $C^{2}$ of $B_{2}$ and to define the set $\mathcal{P}$ for $B_{2}$ by those points inside $B_{2}$ [27. It is simple but has one disadvantage that the points that are uniformly scattered on $C^{2}$ are usually not very uniform on $B_{2}[26$, 28]. It is therefore of fundamental interest to study how well the methodology agree with experimental observations in this paper. Inspired and 45 motivated by Fang and Wang (1994) and Fei et al. (2015), we have as main objectives to truly investigate and gauge whether the bubble swarms are uniformly scatted within a circular region and to present an analysis of computer experiments and real obtained imaging data of the direct-contact boiling heat transfer process.

\section{Experiment and method}

\subsection{Experimental study and image analysis}

This investigation is focused on the bubble evaporation; the present system is based the on non-isothermal DCHE. It has many advantages such as relative simplicity of design, fewer scaling problems, higher heat transfer coefficient (about 20-100 times that of single-phase flow [29]), having no metallic heat transfer surfaces that are prone to corrosion and fouling, and the capacity to operate at relatively small temperature driving forces $\underline{3}$. Direct-contact heat transfer process the exchange of heat between the continuous phase, heat transfer fluid (HTF), and the dispersed phase, R-245fa (1,1,1,3,3 pentafluoropropane), by

${ }_{55}$ bring them into contact directly at different temperature. The apparatus for DCHE is illustrated in Fig 1 , and the mixing uniformity measurements were obtained from the DCHE. Intuitively, the DCHE (1) consists of level gauge (2), viewing 
windows (3), pressure gauge (4), thermometer (5), K-type thermocouple (6) and nozzle (7). The continuous phase was injected into the exchanger at the beginning of our experiment. HTF was heated to the desired temperature that is high enough to make the dispersed phase evaporate completely. After that, the dispersed phase liquid was supplied to DCHE 6o through a tube from a supply tank. Then, the continuous and dispersed phases undergo heat exchange inside the DCHE. The heat transfer process progressively reduces the temperature of R-245fa. The evaporation was allowed to take place until the heat transfer temperature difference had not been enough to cause the phase change of R-245fa. To carried out the experiment, four different parameters, including the height of HTF, the initial heat transfer temperature difference, and the low rate of refrigerant and HTF, were selected. The bubble patterns were imaged using a high-speed shutter video camera 65 (the brand of the video camera was PRAKTICA from Germany and the resolution of the images was 4 million pixels with no light-emitting diode light). For this current analysis, we focused on the properties of mixing transient. The original images are converted to binary images, with black bubbles surrounded by the other nonblack spacing (see Fig. 1p).

\subsection{Measures of uniformity within a circular region}

There are a number of existing methods in the literature to define uniformity of point distribution[26]. Discrepancy is a well-known statistical measure of point set uniformity. Although various definitions of the definitions of the discrepancy measures exist in the literature, discrepancy generally provides a single number that define the overall quality of a set of of sample points. A large discrepancy indicates deviations from uniformity such as clustering or heterogeneity, while small discrepancy indicates that the pattern is more regular. Generally speaking, if a set of $n$ points is uniformly scattered on a unit disk $B_{2}=\left\{(x, y): x^{2}+y^{2} \leq 1\right\}$, the polar coordinates could be used previously:

$$
\left\{\begin{array}{l}
x=r \cos (2 \pi \theta), \\
y=r \sin (2 \pi \theta),
\end{array}\right.
$$

where $(r, \theta) \in C^{2}=[0,1]$. In the following discussions, point-set $\mathcal{P}=\left\{n_{i}\right\}_{i}^{N}$ denotes the $N$ sample points in an arbitrary 70 bounded two-dimensional Euclidean region of interest $\Omega$. For one rectangular local region $[0, \theta] \times[0, r]$, the number of points encapsulated can be counted. The true area of a local region is given by product $\theta r$ and the local discrepancy function $d_{\mathcal{P}}^{*}(\theta, r)$ in this region is given by:

$$
d_{\mathcal{P}}^{*}(\theta, r)=\left|\frac{\mathcal{P} \cap([0, \theta] \times[0, r])}{N}-\theta r\right|
$$

As shown for example in the bottom of Fig 2 it is obvious that these points are not scatted uniformly on $B_{2}$ and that most of these points are concentrated around the center of the $B_{2}$. It is also seen from the top of Fig 2 that the points are scattered quite uniformly. However, a faulty judgment was obtained by the previous method reported in the literature (Fei et al., 2015) obviously. Studying carefully its reason, it is immediately clear from Eq2 2 that the star discrepancy is only applicable to rectangular region $\Omega$. This limitation will inhibit its use in new application areas where the region may not be rectangular 27. If the fan-shaped region is used to replace the rectangle in the definition of $F$-discrepancy, the Eq 3 gives a statistical measure for uniformity of $\mathcal{P}_{F}$ on $B_{2}$.

$$
d_{\mathcal{P}}^{*}(r, \theta)=\left|\frac{N\left(G, \mathcal{P}_{F}\right)}{N}-\frac{v(G)}{u\left(B_{2}\right)}\right|=\left|\frac{N\left(G, \mathcal{P}_{F}\right)}{N}-\frac{\pi r \theta}{\pi}\right|=\left|\frac{N((r, \theta), \mathcal{P})}{N}-r \theta\right|
$$

where $N((r, \theta), \mathcal{P})$ is used to denote the number points in $\mathcal{P}$ falling inside the rectangle $[0, r] \times[0, \theta]$ which is equal to the number of the points $\mathcal{P}_{F}$ belonging to the fan-shaped region as follows,

$$
G=\{(x, y): x=\tilde{q} \cos (2 \pi \phi), y=\tilde{q} \sin (2 \pi \phi), 0 \leq \tilde{q} \leq \sqrt{r}, 0 \leq \phi \leq \theta\}
$$




\section{Results and discussion}

\subsection{Simulation data}

In order to assess the performance of the implementation of proposed scheme, randomly generated small sets were used. Fig 3 is an example of a generated bubbles pattern. As shown in Fig 3 , the black unit square denotes a portion of the mixed zone, the red unit circle denotes the viewing window region, and the green square simulates the image obtained by digital camera. Comparing the discrepancy values of three regions with different shape in Table 1 . It is concluded that star discrepancy methods in rectangle region and circular region have the different accuracy. For the circular shape so of observation region, star discrepancy method in circular-shaped region could outperform star discrepancy method in rectangle-shaped region and perform more sensitive for practical engineering application in some sense. To describe more quantitatively throughout the relative error. From Table 1 , the relative errors of the first parameter mean are $53.68 \%$ and $16.72 \%$, respectively. For the second parameter median, the relative errors are $58.17 \%$ and $13.84 \%$, respectively. Simply, the percentage of enhancing achieved is about $40 \%$.

\section{3.2. Recognition of different uniformity}

The plots in Fig 4 are obtained in non-uniform and uniform flow field, respectively. It is noticed that the two experimental cases have the same Betti numbers $\beta_{1}=151$, which was used to identify different mixing uniformity 20]. However, 151 bubbles distribute in different places. Comparisons results show that the different experimental cases with the same Betti numbers can be identified by this proposed approach.

\section{3.3. Sensitivity analysis}

In order to study the uniformity difference between single bubbles and total bubbles (including bubble swarms) in one bubbles pattern, the proposed approach could be employed. As shown in Fig 5 a, the real bubbles pattern consists of single bubbles and bubble swarms. The most commonly used measure of circularity of objects (i.e., bubbles in this current research) is shown as follows 30 ,

$$
\text { metric }=\frac{4 \pi * \text { area }}{\text { perimeter }^{2}}
$$

By means of the above formula Eq 5 the resulting values were obtained and Fig 5 p presents the circularity of bubbles for the 239 objects in Fig 5 . General speaking, if metric $\geq 0.94$, then the object could be regarded as a disk ( i.e., single bubble). Fig 5 shows the considered single bubbles, with the proportion of $40.17 \%$. It can be seen that the circularity of bubbles does not have a significant influence on the results if adequate bubbles are obtained.

\section{4. Conclusions}

Mathematical processing of bubble swarm images can be can alternative tool to determine the state of homogeneity from a circular-shaped observation region. In this work, the influence of shape of the observation region on measurement of bubble uniformity in a direct-contact heat exchanger was investigated. The difference between rectangular or square region and circular region was discussed. In general, it can be concluded that a small discrepancy denotes uniformly distributed bubble patterns, while a large discrepancy indicates poorly distributed patterns. The space-time features of the mixing transient have been successfully derived when the bubble swarms distribute in a circular region. This work brings new insights to evaluate mixing quality of different systems practically, which has a high degree of accuracy (the percentage of enhancing achieved is about 40\%). Combining statistical information makes it possible to quantify transient aggregates, which are present in circular regions or cubes. Despite our focus on heat transfer process, the statistical image processing framework 105 developed here can be further extended and adapted to study the identification of developmental aggregates. 


\section{Acknowledgements}

We acknowledge the financial support from National Natural Science Foundation of China (Project Nos.: 51666006 \& 51406071) and Scientific and Technological Leading Talent Projects in Yunnan Province (Project No.: 2015HA019). The authors wish to especially thank the referees for numerous detailed questions and comments that greatly improved the 110 presentation. The authors also thank very much Prof. Wuqiang Yang (The University of Manchester), Dr. J. Huang (Yunnan Agricultural University), Dr. S. Wang (Kunming University of Science and Technology) and Dr. Z. Ge (Tsinghua University) for essential help.

\section{References}

[1] S. Kar, X. D. Chen, M. I. Nelson, Direct-contact heat transfer coefficient for condensing vapour bubble in stagnant liquid pool, Chemical Engineering Research \& Design 85 (3) (2007) 320-328.

[2] J. Xu, Q. Xiao, Y. Fei, S. Wang, J. Huang, Accurate estimation of mixing time in a direct contact boiling heat transfer process using statistical methods, International Communications in Heat \& Mass Transfer 75 (2016) $162-168$.

[3] P. Zhang, Y. Wang, C. Guo, K. Wang, Heat transfer in gas-liquid-liquid three-phase direct-contact exchanger, Chemical Engineering Journal 84 (3) (2001) 381-388.

[4] C. P. R. Jr, P. L. C. Lage, Direct-contact evaporation in the homogeneous and heterogeneous bubbling regimes. part I: experimental analysis, International Journal of Heat \& Mass Transfer 47 (17-18) (2004) 3825-3840.

[5] J. Huang, J. Xu, X. Sang, H. Wang, W. Hua, Quantifying the synergy of bubble swarm patterns and heat transfer performance using computational homology, International Journal of Heat \& Mass Transfer 75 (4) (2014) $497-503$.

[6] Y. Fei, Q. Xiao, J. Xu, J. Pan, S. Wang, H. Wang, J. Huang, A novel approach for measuring bubbles uniformity and mixing efficiency in a direct contact heat exchanger, Energy 93 (2015) 2313-2320.

[7] D. Cheng, X. Feng, J. Cheng, C. Yang, Numerical simulation of macro-mixing in liquid-liquid stirred tanks, Chemical Engineering Science 101 (14) (2013) 272-282.

[8] S. Kim, A. N. Nkaya, T. Dyakowski, Measurement of mixing of two miscible liquids in a stirred vessel with electrical resistance tomography, International Communications in Heat \& Mass Transfer 33 (9) (2006) 1088-1095.

[9] F. Diele, F. Notarnicola, I. Sgura, Uniform air velocity field for a bioventing system design: some numerical results, International Journal of Engineering Science 40 (11) (2002) 1199-1210.

[10] J. Favier, A. Revell, A. Pinelli, Numerical study of flapping filaments in a uniform fluid flow, Journal of Fluids \& Structures 53 (1) (2015) 26-35.

[11] Q. Xiao, J. Pan, J. Xu, H. Wang, Z. Lv, Hypothesis-testing combined with image analysis to quantify evolution of bubble swarms in a direct-contact boiling heat transfer process, Applied Thermal Engineering 113 (2017) 851-857.

[12] Y. Yang, Z. Jia, J. Liu, Q. Li, Effect of electric field distribution uniformity on electrospinning, Journal of Applied Physics 103 (10) (2008) 104307-104307-11.

[13] D. Hao, X. Wu, T. Sun, L. Bing, J. Fang, L. Chao, Z. Gao, Effects of electric field intensity and distribution on flame propagation speed of $\mathrm{CH}_{4} / \mathrm{O}_{2} / \mathrm{N}_{2}$ flames, Fuel 158 (2015) 807-815. 
${ }_{140}$ [14] K. Wang, Q. Wang, C. Wang, E. Wang, C. Liu, J. He, Formation of aligned two-phase microstructure in fe-0.25massfields, Materials Letters 62 (10) (2008) 1466-1468.

[15] K. Wang, Q. Wang, C. Wang, Y. Yuan, C. Liu, J. He, Mechanism of formation of aligned two-phase microstructure in a fe-0.25wtfield gradients, Philosophical Magazine Letters 89 (12) (2009) 695-700.

[16] C. Xie, H. Zhang, L. J. Shimkets, O. A. Igoshin, Statistical image analysis reveals features affecting fates of Myxococcus xanthus developmental aggregates, Proceedings of the National Academy of Sciences of the United States of America 108 (14) (2011) 5915-5920.

[17] S. Karami, M. Imani, F. Farahmandghavi, A novel image analysis approach for evaluation of mixing uniformity in drug-filled silicone rubber matrix, International Journal of Pharmaceutics 460 (1-2) (2014) 158-64.

[18] J. Aubin, M. Ferrando, V. Jiricny, Current methods for characterising mixing and flow in microchannels, Chemical Engineering Science 65 (6) (2010) 2065-2093.

[19] M. Gameiro, K. Mischaikow, T. Wanner, Evolution of pattern complexity in the Cahn-Hilliard theory of phase separation, Acta Materialia 53 (3) (2005) 693-704.

[20] J. Xu, H. Wang, H. Fang, Multiphase mixing quantification by computational homology and imaging analysis, Applied Mathematical Modelling 35 (5) (2011) 2160-2171.

155 [21] J. Xu, Q. Xiao, Y. Chen, Y. Fei, J. Pan, H. Wang, A modified L $_{2}$-star discrepancy method for measuring mixing uniformity in a direct contact heat exchanger, International Journal of Heat \& Mass Transfer 97 (2016) 70-76.

[22] Q. Xiao, J. Xu, H. Wang, Quantifying the evolution of flow boiling bubbles by statistical testing and image analysis: toward a general model, Scientific Reports 6 (2016) 31548.

[23] H. Niederreiter, Low-discrepancy and low-dispersion sequences, Journal of Number Theory 30 (1) (1988) 51-70.

[24] D. P. Dobkin, D. Eppstein, D. P. Mitchell, Computing the discrepancy with applications to supersampling patterns, Acm Transactions on Graphics 15 (4) (1996) 354-376.

[25] Y. Saka, M. Gunzburger, J. Burkardt, Latinized, improved lhs, and CVT point sets in hypercubes, International Journal of Numerical Analysis \& Modeling (3-4) (2007) 729-743.

[26] S. O. Meng, C. K. Ye, P. L. Ooi, Statistical measures of two dimensional point set uniformity, Computational Statistics \& Data Analysis 56 (6) (2012) 2159-2181.

[27] K. Fang, Y. Wang, Number-theoretic methods in statistics, Chapman \& Hall, 1994.

[28] A. Petrie, T. R. Willemain, An empirical study of tests for uniformity in multidimensional data, Computational Statistics \& Data Analysis 64 (4) (2013) 253-268.

[29] Y. Li, C. Liu, Z. Shen, Heat transfer in bubble column and gas-lift loop reactors, Journal of Engineering Thermophysics 10 (1) (1989) 72-74.

[30] G. J. Awcock, R. Thomas, Applied Image Processing, Macmillan Education UK, 1995. 


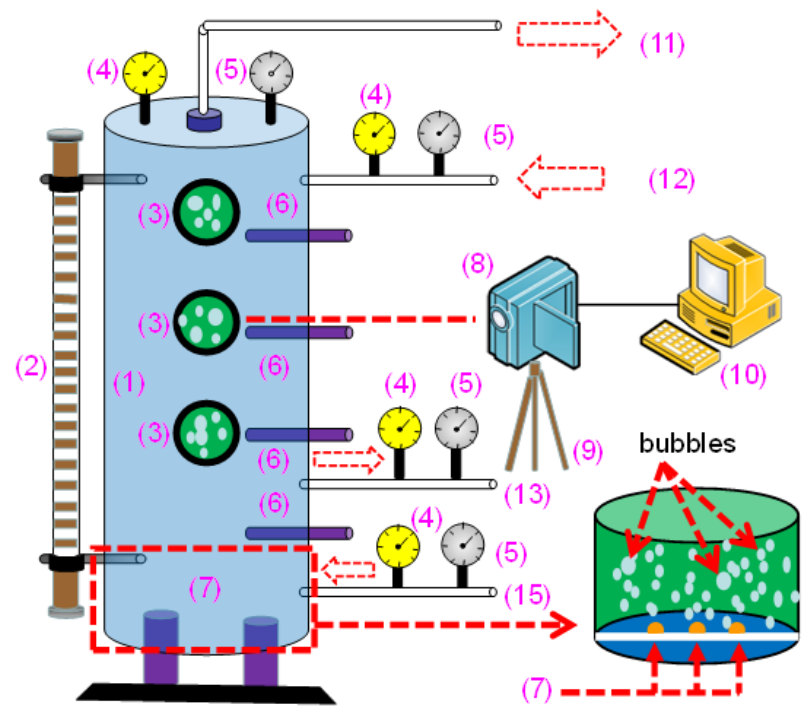

(a)

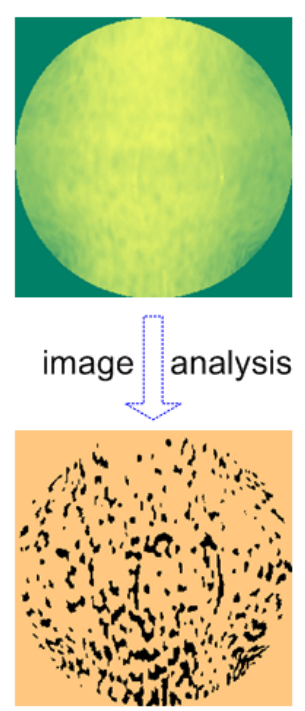

(b)

Figure 1: (a) Schematic illustration of DCHE used for the work presented here; (b) Treatment for one piece of bubble swarm patterns reported by Ref.[21]. 


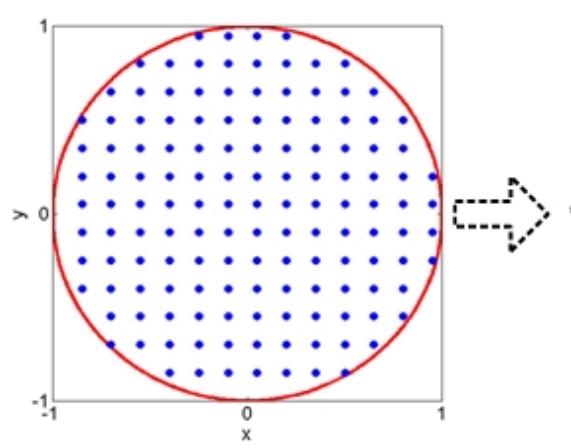

orthogonal coordinates

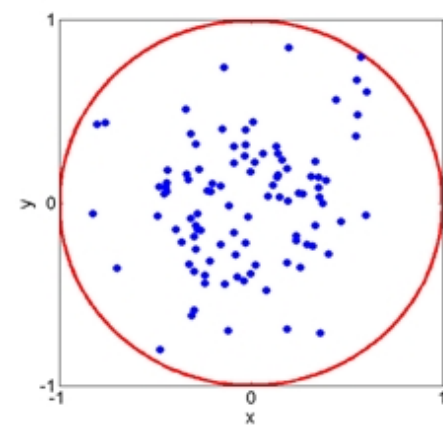

orthogonal coordinates

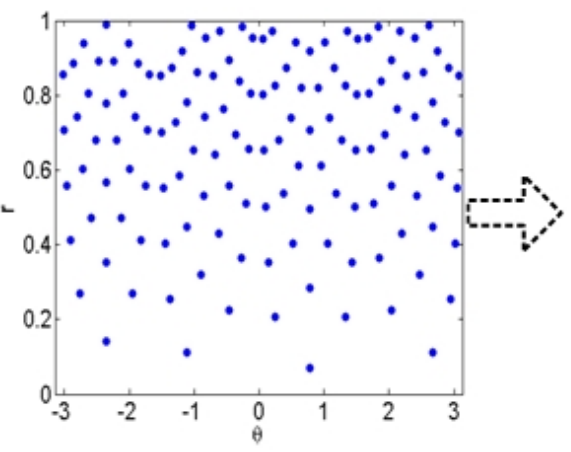

polar coordinates

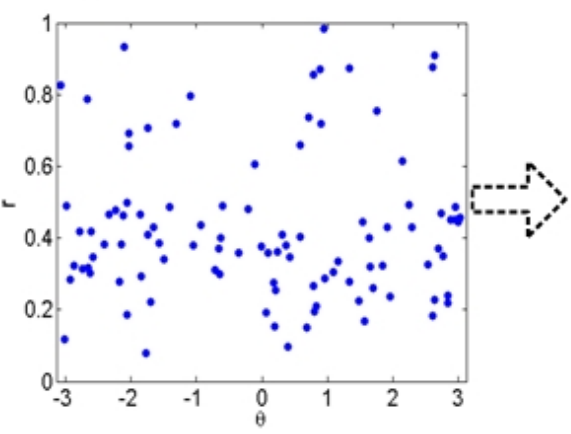

polar coordinates

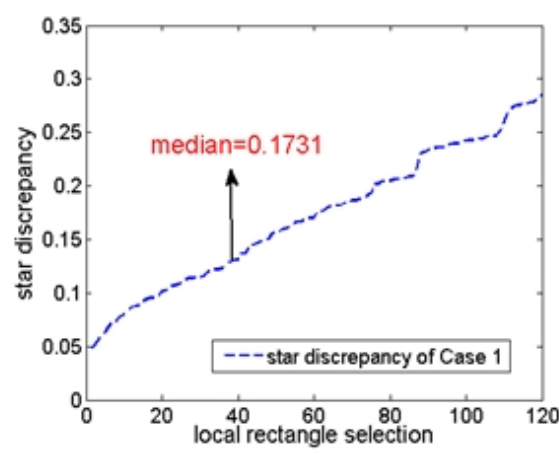

star discrepancy

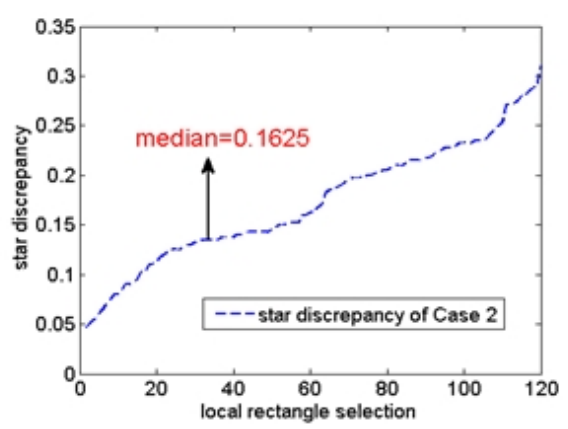

star discrepancy

Case 1

Case 2

Figure 2: Two sets of points (i.e., Case 1 and Case 2) on $B_{2}=\left\{(x, y): x^{2}+y^{2} \leq 1\right\}$. 


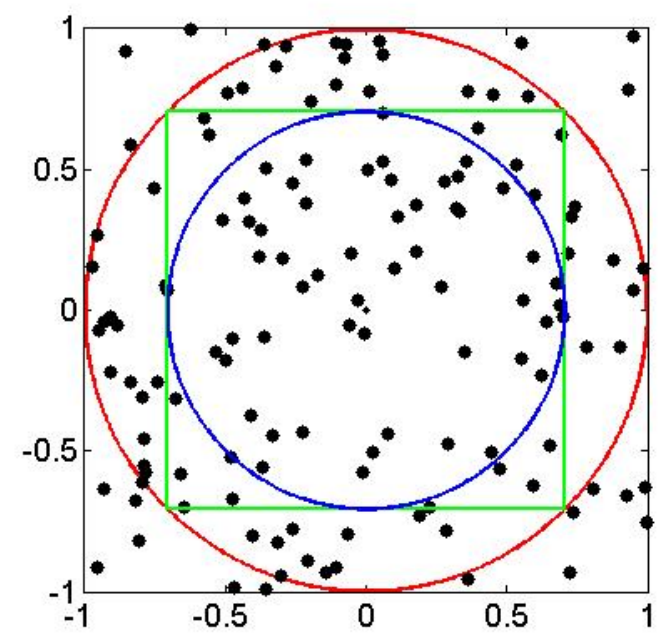

Figure 3: Computer-generated bubble pattern group of random distribution for testing. 


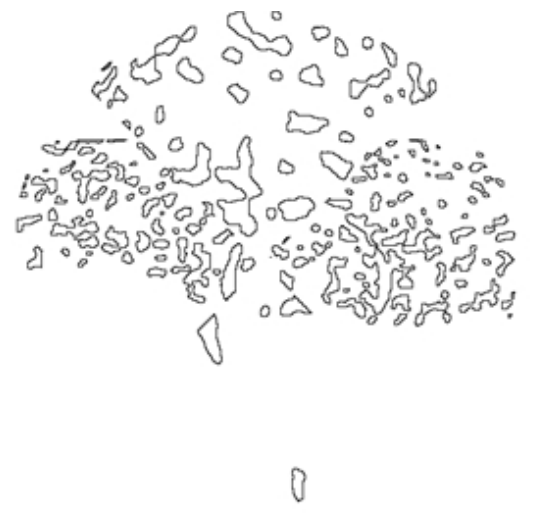

(a)

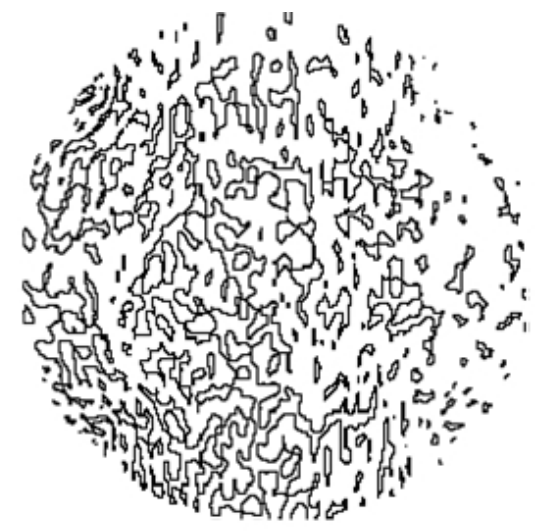

(b)

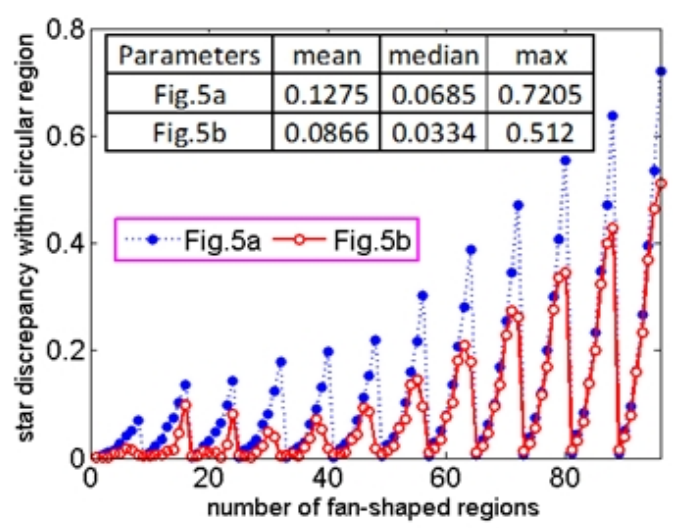

(c)

Figure 4: Experimental bubble pattern in worse case (a) and better case (b); (c) recognition of different mixing efficiency (considering three parameters). 


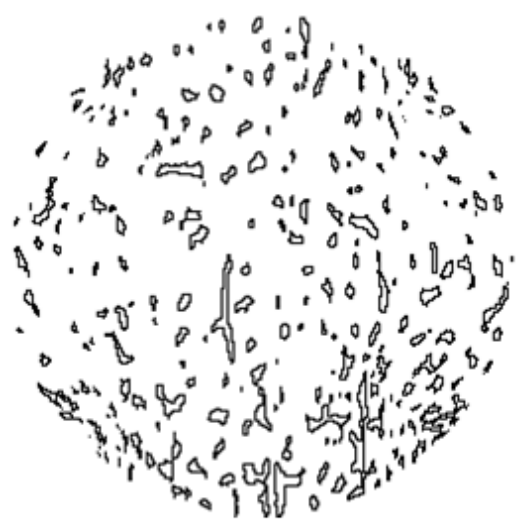

(a)

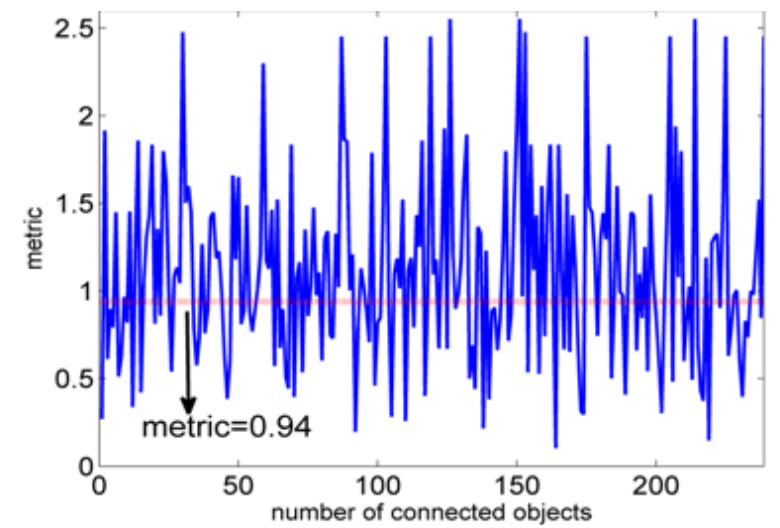

(b)

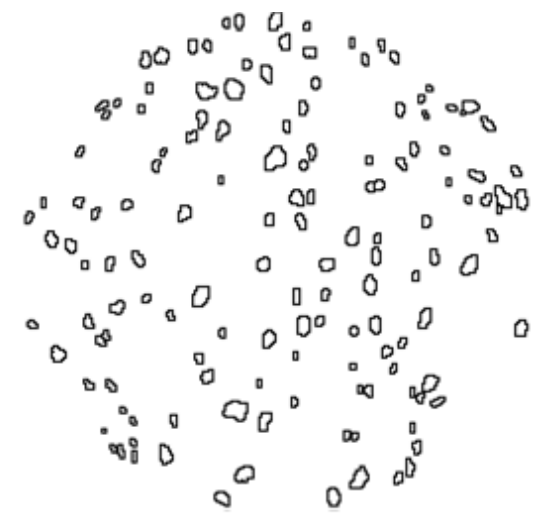

(c)

Figure 5: Sensitivity analysis: (a) original bubbles pattern $\left(d_{\text {total }}^{*}:\right.$ mean $=0.0714$, median $\left.=0.0221, \max =0.4917\right)$; (b) circularity of 239 objects in the original bubbles pattern; (c) the considered single bubbles $\left(d_{\text {disk }}^{*}:\right.$ mean $=0.0 .0742$, median $\left.=0.0210, \max =0.5385\right)$. 
Table 1: Computing results of mixing uniformity by the star discrepancy method in rectangle-shaped and circular-shaped regions.

\begin{tabular}{c|c|c|c}
\hline Parameters & red unit circle & green square & blue inscribed circle \\
\hline mean & $d_{11}^{*}=0.1507$ & $d_{21}^{*}=0.0698$ & $d_{31}^{*}=0.1255$ \\
median & $d_{12}^{*}=0.1236$ & $d_{22}^{*}=0.0517$ & $d_{32}^{*}=0.1065$ \\
$\max$ & $d_{13}^{*}=0.5072$ & $d_{23}^{*}=0.5072$ & $d_{33}^{*}=0.5072$ \\
\hline
\end{tabular}

